\title{
Apoiando a colaboração de recém-chegados em equipes de desenvolvimento de software
}

\author{
João Paulo B. Glória' ${ }^{1,2}$, Adriana S. Vivacqua ${ }^{1}$ \\ ${ }^{1}$ Programa de Pós-Graduação em Informática - Instituto de Computação - Universidade \\ Federal do Rio de Janeiro (UFRJ) \\ Rio de Janeiro - RJ - Brasil \\ ${ }^{2}$ Instituto Federal de Educação, Ciência e Tecnologia Baiano (IF Baiano) \\ Guanambi - BA - Brasil \\ joao.gloriaeifbaiano.edu.br, avivacquaedcc.ufrj.br
}

\begin{abstract}
The newcomers onboarding process into software development teams depends heavily on well-defined processes and collaboration between the novice and his mentors. An effective integration allows newcomers to have access to the people and information needed to perform their work more quickly. This research goals to investigate the onboarding process of newcomers into software development teams. The proposed methodology aims to understand the strategies and barriers in the onboarding process, besides, we propose to create an intelligent agent model to enable better collaboration and integration of newcomers in software development teams.
\end{abstract}

Resumo. O processo de integração de recém-chegados em equipes de desenvolvimento de software depende fortemente de processos bem definidos e da colaboração entre o novato e seus mentores. Um processo de integração eficaz dá aos novos membros, acesso a pessoas e informações necessárias para desempenhar suas funções com mais rapidez. O objetivo geral da pesquisa é investigar o processo de integração de recém-chegados em equipes de desenvolvimento de software. A metodologia proposta visa entender as estratégias e barreiras no processo de integração, além disso, será proposta a criação de um modelo de agente inteligente para viabilizar a colaboração e integração de recém-chegados em equipes de desenvolvimento de software.

\section{Introdução}

Um dos grandes desafios encontrados por empresas de software é a chegada de novos membros nas equipes de desenvolvimento (SNELL, 2006). A chegada de um novato pode impactar a produtividade da empresa de uma forma indireta, e pode também impactar diretamente nas rotinas da equipe e das pessoas designadas a auxiliá-lo. A inclusão de novos membros em um projeto tem se mostrado altamente benéfica no longo prazo, porém diminui a produtividade da equipe no curto prazo (VIVIANI; MURPHY, 2019).

As empresas estão constantemente aprimorando seus processos e técnicas para melhorar a produtividade de suas equipes. Um dos principais problemas de produtividade é a rotatividade de membros das equipes. Além disso, parte do processo de integração de um novato envolve alocar um "amigo", um "líder" ou "mentor" que mostrará ao recémchegado como funciona a equipe e também como operar no ambiente de trabalho (SNELL, 2006). 
Ao se tratar de equipes de desenvolvimento de software, o desafio é grande pois é necessário que os recém-chegados se familiarizem rapidamente não só com a empresa, mas também com as especificidades dos projetos e os recursos técnicos necessários para o trabalho. Obter uma compreensão dos recursos relevantes em um novo projeto é vital para a integração bem-sucedida de um recém-chegado. No entanto, isso geralmente não é fácil e requer tempo e esforço até que os recém-chegados se familiarizem com os recursos do projeto e superem as possíveis dificuldades encontradas. (WANG; SARMA, 2011)

Um processo de integração eficaz permite que novos membros da equipe tenham acesso às pessoas, informações, ferramentas e materiais necessários para desempenhar suas funções com mais rapidez. Um processo de integração bem projetado e automatizado reduz custos para a empresa, reduz o tempo da primeira contribuição do novato, aumenta a produtividade e o desempenho dos funcionários, gera vínculos mais fortes entre colegas com maior satisfação e lealdade no trabalho (SNELL, 2006). Além disso, com uma integração feita de forma a garantir uma maior homogeneidade do grupo, gera uma maior sinergia, que ajuda fortemente nos resultados da empresa (MOSER; ARAÚJO, 2020).

Atualmente o processo de integração de um recém-chegado em uma equipe de desenvolvimento de software depende fortemente de processos e pessoas, como por exemplo, o trabalho dos mentores que são fundamentais para uma boa integração do novato. Dessa forma, é um processo que requer colaboração entre o novato e membros mais experientes das equipes. No entanto, para promover a integração de novatos nas equipes, alguns recursos computacionais são utilizados, como por exemplo, a utilização de bots, aplicativos, ferramentas, sites, portais e e-mails (DOMINIC; RITTER; RODEGHERO, 2020; JENSEN; KING; KUECHLER, 2011; MORGAN et al., 2013).

Os aplicativos de mensagens instantâneas têm sido amplamente adotados como uma das principais tecnologias de comunicação e troca de informações. Atualmente, a maioria deles oferece suporte para a integração de outros aplicativos, como por exemplo, os chatbots (DANIEL et al., 2020). Chatbots são agentes inteligentes com os quais os usuários podem manter conversas, seja por texto ou voz (SÁNCHEZ-DÍAZ et al., 2018). O chatbot é um subtipo de um software bot, no qual o objetivo é simular uma conversa humana. Ele é o resultado da ligação entre os usuários e interfaces computacionais modernas (REYES et al., 2020).

Nos últimos anos, os chatbots se tornaram populares em empresas voltadas para o atendimento ao cliente (SÁNCHEZ-DÍAZ et al., 2018), assim como em empresas da área de educação, e-commerce e nas redes sociais (DANIEL et al., 2020). As principais funções dos chatbots estão relacionadas a função de vendas $(41 \%)$, seguido por suporte (37\%), marketing (17\%) e outros (5\%). A previsão de crescimento para o mercado de assistentes virtuais é de US $\$ 8,9$ bilhões de dólares até 2025. Os usuários dizem que os chatbots são melhores porque estão disponíveis 24 horas por dia, 7 dias por semana, reduzem os tempos de espera e são rápidos no encaminhamento para a equipe certa (Forbes, 2019). Desta forma, com a evolução dos chatbots abrem-se as portas para aprimorar o processo de integração dos recém-chegados.

O objetivo geral da pesquisa é investigar o processo de integração de recémchegados em equipes de desenvolvimento de software de modo a propor soluções para melhorar a colaboração entre o recém-chegado e a equipe. Conforme Gil (2010), toda 
pesquisa se inicia com algum tipo de problema ou indagação. Para descrever o problema de pesquisa deste trabalho a questão de pesquisa é formulada da seguinte forma:

\section{“Como apoiar a colaboração na integração de recém-chegados em equipes de desenvolvimento de software?"}

Para abordar esta pergunta e alcançar o objetivo deste trabalho, foram definidas três questões de pesquisa específicas:

QP1. Quais são as barreiras enfrentadas pelos recém-chegados em equipes de desenvolvimento de software?

QP2. Quais são as práticas ou estratégias utilizadas para integração dos recém-chegados em equipes de desenvolvimento de software?

QP3. Como a utilização de um agente inteligente pode facilitar a colaboração de novatos em equipes de desenvolvimento de software?

\section{Revisão bibliográfica}

\subsection{Integração de recém-chegados}

O processo no qual um funcionário novo chega em uma empresa sem nenhum conhecimento e passa a ser um funcionário totalmente funcional e independente é chamado de socialização organizacional. Pode-se encontrar este tema na literatura por integração, internalização e amplamente conhecido pelo termo em inglês 'onboarding'. A integração é o processo pelo qual o novo funcionário deixa de ser um estranho na empresa e passa a se tornar um membro funcional. Desta forma, a integração engloba todo processo necessário para que o novato aprenda os conhecimentos, habilidades e competências necessárias para ter êxito na nova empresa. (BAUER; ERDOGAN, 2010)

O processo de integração é importante tanto para o funcionário, quanto para as empresas. Uma das maneiras pelas quais as empresas podem ajudar a criar funcionários engajados é por meio da integração eficaz. Essa integração poderá resultar em funcionários eficientes, com perspectiva de trabalho positiva, o que permitirá uma permanência de tempo maior na empresa. Por outro lado, um processo de integração ruim poderá levar a saída prematura daquele funcionário, ou até mesmo a ineficácia do trabalho. Isso faz com que o ciclo de recrutamento e seleção de funcionários seja reiniciado, levando a perda de tempo e recursos financeiros. (BAUER; ERDOGAN, 2010)

Todos os novos funcionários passam por uma curva de aprendizado durante a qual seu desempenho é inferior ao de um funcionário totalmente integrado. As curvas de aprendizagem dos funcionários representam o tempo necessário para que os funcionários em novos cargos atingem a produtividade total e a taxa na qual eles progridem em direção à produtividade total. $\mathrm{O}$ fim do processo de integração se encerra quando o aprendizado do novo funcionário se equivale ao nível de conhecimento dos demais funcionários. (SNELL, 2006)

Os problemas que são enfrentados durante a curva de aprendizado são chamados de barreiras. Elas podem ser analisadas sob diversas perspectivas, como por exemplo, as barreiras que afetam diretamente o recém-chegado, as barreiras que afetam diretamente o mentor responsável em auxiliá-lo e também as barreiras que afetam a equipe. Essas 
barreiras podem estar relacionadas a problemas técnicos, falta de conhecimento e falta de documentação, porém a maioria das barreiras são sociais, ou seja, de colaboração e relacionamento com demais integrantes da equipe. (BALALI et al., 2018)

Já os benefícios do processo de uma integração eficaz são: o acolhimento do grupo ao recém-chegado, a clara distribuição dos papéis e responsabilidades dos integrantes do time, a diminuição de problemas, uma maior integração da equipe, maior produtividade, melhor divisão da carga de trabalho, melhoria nas entregas, maior motivação, maior segurança, sentimento de pertencimento pelo recém-chegado e tempo hábil para o aprendizado (MOSER; ARAÚJO, 2020).

\subsection{Agentes inteligentes}

Um agente é um sistema de computador que deve ser capaz de perceber o ambiente em que está localizado a fim de atender aos seus objetivos de design. Ele deve ser autônomo, ou seja, ser capaz de agir sem a intervenção direta de humanos e deve ter controle sobre suas próprias ações. Os agentes devem ser flexíveis o que os proporciona a capacidade de serem responsivos, proativos e que interagem com humanos ou com outros agentes. (SRIVASTAVA, 1999)

Os chatbots são um tipo de agente inteligente com os quais os usuários podem manter conversas, que geralmente são por texto ou voz (SÁNCHEZ-DÍAZ et al., 2018). A ideia inicial do chatbot era ver se esses tipos de sistemas podiam enganar os usuários, dizendo que eram humanos reais. O seu objetivo era então simular uma conversa humana, ou seja, emular a comunicação de chat informal entre um usuário humano e um computador usando a linguagem natural. No entanto, esses sistemas não foram construídos apenas para imitar a conversa humana e entreter os usuários. Eles são construídos para viabilizar o uso da linguagem natural para se comunicar com os computadores (ABU SHAWAR; ATWELL, 2007).

Os chatbots se distinguem do domínio maior dos bots de software por sua capacidade de usar a linguagem para se comunicar com seus usuários. Termos diferentes têm sido utilizados para descrever um chatbot, como: sistema de conversação de máquina, entidades conversacionais artificiais, agente virtual, talkbots, sistema de diálogo e chatterbot. Esses tipos de bots podem ter seus próprios serviços internos ou acesso a serviços externos (LEBEUF, 2018). Esses tipos de bots possuem identidades e personalidades humanas para tornar a conversa mais natural. (SÁNCHEZ-DÍAZ et al., 2018). A melhor maneira de facilitar a Interação Humano-Computador (IHC) é permitindo que os usuários expressem seus interesses, desejos ou consultas de forma direta e natural, falando, digitando e apontando. Este foi $\mathrm{o}$ impulsionador do desenvolvimento dos chatbots (ABU SHAWAR; ATWELL, 2007).

Apesar de cada bot ter seus próprios detalhes e limitações de implementação, a maioria das ferramentas deriva de uma ideia geral, onde deve-se receber dados brutos, dar-lhes significado e, então, agir apropriadamente de acordo com uma base de conhecimento. Para processar a linguagem natural, esses bots contam com o reconhecimento de padrões. No entanto, cabe aos designers do software gerar a base de conhecimento e fornecer ao mecanismo de aprendizagem, exemplos apropriados ao domínio (SÁNCHEZ-DÍAZ et al., 2018). Entre os milhares de chatbots que existem hoje, alguns exemplos populares incluem: Siri da Apple, Cortana da Microsoft, Alexa da Amazon, Google Assistant e Watson da IBM. (LEBEUF, 2018) 


\section{Metodologia de pesquisa}

Nesta seção serão abordados os métodos de pesquisa utilizados e como eles serão estruturados em busca de responder às questões de pesquisa apresentadas neste trabalho. De forma a responder às questões de pesquisa, a análise será realizada em abordagem qualitativa e quantitativa. Para melhor compreensão, este estudo será dividido em três etapas, ilustradas na Figura 1. Cada etapa visa responder a uma questão de pesquisa específica. Na primeira etapa será realizada uma revisão sistemática da literatura (RSL) para identificar e entender quais são as estratégias e as barreiras enfrentadas pelos recémchegados em uma equipe de desenvolvimento de software.

Muitas das barreiras enfrentadas pelos recém-chegados não estão identificadas na literatura (BALALI et al., 2018; STEINMACHER, 2015), desta forma, nesta segunda etapa será feita uma análise através de entrevistas semiestruturadas com diversos desenvolvedores de software com o objetivo de ampliar o conhecimento sobre estratégias e as barreiras que porventura não estejam listadas na literatura, enfocando em particular questões associadas à colaboração. Assim, após a conclusão da segunda etapa, será possível responder às questões de pesquisa QP1 e QP2.

\begin{tabular}{|c|c|c|c|}
\hline \multicolumn{4}{|c|}{ Desenho de pesquisa } \\
\hline $1^{2}$ Etapa & $2^{\circ}$ Etapa & & \\
\hline $\begin{array}{c}\text { Revisão sistemática da } \\
\text { literatura sobre } \\
\text { estratégias e barreiras }\end{array}$ & $\left(\begin{array}{c}\text { Estudo qualitativo } \\
\text { com entrevistas para } \\
\text { entender as } \\
\text { estratégias e } \\
\text { barreiras }\end{array}\right.$ & $\begin{array}{l}\text { Definição de um } \\
\text { modelo de agente } \\
\text { inteligente para } \\
\text { superar as barreiras }\end{array}$ & $\begin{array}{l}\text { Estudo com base em } \\
\text { experimento para } \\
\text { avaliar o modelo de } \\
\text { agente inteligente }\end{array}$ \\
\hline
\end{tabular}

Figura 1 - Metodologia de pesquisa proposta

$\mathrm{Na}$ terceira e última etapa, será proposta a criação de um modelo de agente inteligente nos moldes de um chatbot que visa permitir a integração e colaboração de recém-chegados em equipes de desenvolvimento de software. Porém, para responder a QP3 será necessário realizar um experimento com os recém-chegados para avaliar o modelo proposto. Para avaliação do experimento, questionários (survey) e entrevistas poderão ser realizados com os participantes do experimento.

Com esta pesquisa espera-se obter um conhecimento aprofundado sobre as barreiras que impedem ou atrapalham o processo de integração de recém-chegados em equipes de desenvolvimento de software. Além disso, é esperado um entendimento das estratégias que são adotadas por equipes e empresas de software para promover a integração dos recém-chegados. Por fim, almeja-se propor uma solução baseada em agentes inteligentes para viabilizar uma melhor colaboração no processo de integração de recém-chegados em equipes de desenvolvimento de software.

\section{Referências bibliográficas}

SHAWAR, Bayan Abu; ATWELL, Eric. Chatbots: are they really useful?. In: Ldv forum. 2007. p. 29-49.

BALALI, Sogol et al. Newcomers' barriers... is that all? an analysis of mentors' and newcomers' barriers in OSS projects. Computer Supported Cooperative Work (CSCW), v. 27, n. 3-6, p. 679-714, 2018. 
BAUER, Talya N.; ERDOGAN, Berrin. Organizational socialization: The effective onboarding of new employees. 2011.

Forbes, 2019. AI Stats News: Chatbots Increase Sales By 67\% But 87\% Of Consumers Prefer Humans. Disponível em: <https://www.forbes.com/sites/gilpress/2019/11/25/aistats-news-chatbots-increase-sales-by-67-but-87-of-consumers-prefer-

humans/?sh=1e4a039048a3>. Acesso em: 10 mar. 2021.

DOMINIC, James; RITTER, Charles; RODEGHERO, Paige. Onboarding Bot for Newcomers to Software Engineering. In: Proceedings of the International Conference on Software and System Processes. 2020. p. 91-94.

DANIEL, Gwendal et al. Xatkit: a multimodal low-code chatbot development framework. IEEE Access, v. 8, p. 15332-15346, 2020.

GIL, A. C. Como elaborar projetos de pesquisa. 5. ed. ed. São Paulo. 2010.

JENSEN, Carlos; KING, Scott; KUECHLER, Victor. Joining free/open source software communities: An analysis of newbies' first interactions on project mailing lists. In: 2011 44th Hawaii international conference on system sciences. IEEE, 2011. p. 1-10.

LEBEUF, Carlene R. A taxonomy of software bots: towards a deeper understanding of software bot characteristics. 2018. Tese de Doutorado.

MORGAN, Jonathan T. et al. Tea and sympathy: crafting positive new user experiences on wikipedia. In: Proceedings of the 2013 conference on Computer supported cooperative work. 2013. p. 839-848.

MOSER, P. C.; ARAÚJO, J. I. DA S. Internalização de novos membros em equipes de desenvolvimento de software: uma versão detalhada. iSys - Brazilian Journal of Information Systems, v. 13, n. 3, p. 25-54, 2020.

REYES, R. et al. Virtual Assistants in Education : Model , Implementation and Results. November, 2020.

SÁNCHEZ-DÍAZ, X. et al. A knowledge-based methodology for building a conversational chatbot as an intelligent tutor. Lecture Notes in Computer Science (including subseries Lecture Notes in Artificial Intelligence and Lecture Notes in Bioinformatics), v. 11289 LNAI, n. March, p. 165-175, 2018.

SNELL, A. Researching onboarding best practice: Using research to connect onboarding. Strategic HR Review, v. 5, n. 6, p. 32-35, 2006.

SRIVASTAVA, S. K. Applications of Intelligent Agents. Electronics Information and Planning, v. 26, n. 5, p. 273-281, 1999.

STEINMACHER, Igor Fábio. Supporting newcomers to overcome the barriers to contribute to open source software projects. 2015. Tese de Doutorado. Universidade de São Paulo.

VIVIANI, G.; MURPHY, G. C. Reflections on onboarding practices in mid-sized companies. Proceedings - 2019 IEEE/ACM 12th International Workshop on Cooperative and Human Aspects of Software Engineering, CHASE 2019. Anais...IEEE, 2019

WANG, J.; SARMA, A. Which bug should I fix: Helping new developers onboard a new project. Proceedings - International Conference on Software Engineering, $n$. November, p. 76-79, 2011. 